\title{
8
}

Research Square

\section{Investigation of a novel implant design for the treatment of reverse oblique femoral intertrochanteric fractures}

\section{Rui Zhang ( $\sim$ orthop_zhangrui@163.com)}

Zhejiang University School of Medicine Fourth Affiliated Hospital https://orcid.org/0000-0003-28358963

\section{Kangyi Wang}

Dongyang People's Hospital

\section{Peng Luo}

The Second Affiliated Hospital of Wenzhou Medical University

\section{Xiaoshan Guo}

The Second Affiliated Hospital of Wenzhou Medical University

\section{Research article}

Keywords: Reverse oblique femoral intertrochanteric fracture, internal fixation, biomechanical test, intramedullary, extramedullary.

Posted Date: October 2nd, 2019

DOI: https://doi.org/10.21203/rs.2.15562/v1

License: (1) This work is licensed under a Creative Commons Attribution 4.0 International License. Read Full License 


\section{Abstract}

Objective: To evaluate the biomechanical properties of intramedullary fixation, lateral plate, medial buttress plate and bilateral plate for fixing reverse oblique intertrochanteric fractures.

Methods: Twenty-four synthetic femoral bone models (Synbone) were divided into four groups [proximal femoral nail anti-rotation (PFNA), proximal femoral medial buttress plate (PFMBP), proximal femoral locking compression plate (PFLCP) and proximal femoral medial buttress plate + proximal femoral locking compression plate (PFMBL+PFLCP)], and an unstable, reverse oblique femoral intertrochanteric fracture was made. After fixation, each model underwent axial load testing, torsion testing, and axial loadto-failure testing. The stiffness of axial and torsion, the torque of different torsion angles and the failure load of every model were recorded.

Results: The bilateral plate showed the best performance in axial load, torsion, and load-to-failure testing. The stiffness of axial and torsion in the PFMBP was superior to the PFNA [162.05 $\pm 22.05 \mathrm{~N} / \mathrm{mm}$ vs. $119.13 \pm 29.14 \mathrm{~N} / \mathrm{mm}$ in axial, $(1.36 \pm 0.32) \mathrm{N} \times \mathrm{mm} / \mathrm{deg}$ vs. $(1.10 \pm 0.13) \mathrm{N} \times \mathrm{mm} / \mathrm{deg}$ in torsion, $\mathrm{P}<0.05]$, whereas there was no significant difference between PFMBP, PFLCP and PFLCP+PFMBP in torsion stiffness [(1.36 \pm 0.32$) \mathrm{N} \times \mathrm{mm} / \mathrm{deg},(1.45 \pm 0.44) \mathrm{N} \times \mathrm{mm} / \mathrm{deg},(1.45 \pm 0.18) \mathrm{N} \times \mathrm{mm} / \mathrm{deg}, \mathrm{P}>0.05]$.

Conclusion: Our newly designed medial buttress plate achieved greater stiffness in axial load and torsion than PFNA and PFLCP to fix reverse oblique intertrochanteric fractures in biomechanical research, whereas the bilateral plate showed better stiffness than PFMBP.

\section{Background}

Reverse oblique intertrochanteric fractures are a particular kind of proximal femoral fracture in which the fracture line extends through the lateral femoral cortex distal to the vastus ridge of the greater trochanteric. Such fractures are classified as 31-A3 according to the Orthopaedic Trauma Association (OTA) classification system, and could be further classified as 31-A3.1 (reverse oblique), 31-A3.2 (transverse), and 31-A3.3 (multi-fragmentary). Reverse oblique intertrochanteric fractures account for nearly $2 \%$ to $23 \%$ of all trochanteric fractures in some epidemiology studies [1-5], and the incidence is increasing with osteoporotic elderly people and young adults involved in traffic accidents and other impact events. These unstable fractures are a challenge to surgeons because of the medialization and shortening of the shaft with angulation and external rotation of the proximal fragment, which is caused by the work of the iliopsoas, adductor, and abductor muscles, generating shear forces across the fracture line. The treatment for reverse oblique intertrochanteric fractures is normally divided into two kinds of fixation methods: intramedullary and extramedullary. Presently, more surgeons choose intramedullary fixation methods such as Gamma3 nail, proximal femoral nail, and proximal femoral nail anti-rotation, instead of extramedullary devices. In addition, some researchers proved that intramedullary methods could provide better biomechanical effects [6,7]. The proximal femur has tension and stress bone trabecular, under tensile stress and compressive stress, respectively. Traditional extramedullary fixation is 
primarily against the tensile stress from lateral cortical bone trabecular, whereas intramedullary fixation is primarily against the shear force and tensile stress from the fracture line. As medial buttress plate has never been used in this type of injury, we hypothesized that the use of this plate to treat reverse oblique femoral intertrochanteric fracture could yield a similar or better outcome. A cadaveric research study proved that a new anterolateral intermuscular approach could provide excellent exposure and less softtissue damage than the traditional approach with the treatment of intertrochanteric fractures [8]. In addition, wide exposure also benefit the medial approach. Because extramedullary devices are primarily located in the lateral of femoral, we asked whether there is a medial device that could treat reverse oblique intertrochanteric fractures in a new approach. On the basis of the aforementioned theories, we designed a new medial plate (proximal femoral medial buttress plate). The purpose of this study was to evaluate the biomechanical properties of intramedullary fixation, lateral plate, and medial buttress plate for fixing reverse oblique intertrochanteric fractures.

\section{Methods}

Our experiment was conducted in the Tianjin Orthopaedic Biomechanical Laboratory (Tianjin Hospital, Tianjin, China), and four implant groups were included: group A: proximal femoral nail anti-rotation (PFNA; Watson Co., Ltd., Changzhou, China); group B: proximal femoral medial buttress plate (made of titanium alloy); group C: proximal femoral locking compression plate (PFLCP; Watson Co., Ltd., Changzhou, China); group D: both proximal femoral medial buttress plate and proximal femoral locking compression plate (PFMBP+PFLCP). Our newly designed T-shape medial buttress plate maintains three holes proximally and eight holes distally, and two lag screws could be planted in the medial of intertrochanteric through two distal holes (Figure 1). The PFLCP (7 holes, left) includes four holes proximally and seven holes distally, with 120 degrees of proximal screws. The PFNA that we chose consisted of a 9-mm distal diameter nail, a length of $340 \mathrm{~mm}$, a 130-degree neck angle, and $10.5 \times 95 \mathrm{~mm}$ anti-rotation blade screws. Two locking screws $(5 \times 50 \mathrm{~mm})$ were inserted into the static locking hole for the PFNA. The Instron E10000 (Instron Corporation, Norwood, MA, USA) dynamic multi-dimensional biomechanical fatigue testing machine was used for the biomechanical test. The Instron E10000 testing machine features up to $\pm 10,000 \mathrm{~N}$ of axial force capacity and $\pm 100 \mathrm{Nm}$ torque capacity. Axial load and rotation data were recorded by Bluehill 2 software (Instron Corporation, Norwood, MA, USA) and MAX software (Instron Corporation, Norwood, MA, USA). The medial buttress plate was placed by first using two Kirschner wires to temporarily fix the fracture. Then, two proximal screws were inserted. Lag screws were inserted after the Kirschner wires were removed, and the remaining screws were then inserted. Normally, two or three screws were used proximally, and five or six screws were used distally. The PFLCP was also placed using two Kirschner wires to first fix the fracture, followed by the insertion of three proximal screws, and then the remaining four or five distal screws. In the bilateral group, the medial buttress plate was planted before the PFLCP, and the location and length of the PFLCP's screws were chosen according to the medial buttress plate's screws. The PFNA was inserted with an intramedullary nail aiming device (Watson Co., Ltd., Changzhou, China). 


\section{Specimens}

Twenty-four synthetic femoral bone models (the 4th generation left adult composite femurs, Malans Synbone, Switzerland) were randomly, average-divided into four groups. They had a length of $337 \mathrm{~mm}, \mathrm{a}$ neck shaft angle of 135 degrees, a femoral head diameter of $48 \mathrm{~mm}$, and an anteversion angle of 15 degrees. All synthetic bones were filled with dense inner foam and a synthetic cortical layer, which simulate cancellous bone.

\section{Model}

To simulate reverse oblique intertrochanteric fractures, osteotomies were created by jig saw in each specimen with an angle of 60 degrees running inferolaterally from the lesser trochanteric. Every fracture line was similar. After the fracture lines were created, all implants were fixed in each model, which was performed by the same researcher (Figure 2). There are no gaps or displacement between the fracture fragments. Every model was embedded in a metal tube using ethoxyline resin (ER) to simulate the singleleg stand of the femur adducted 15 degrees at the frontal plane and vertical at the sagittal plane, maintaining a medial rotation of 5 to 10 degrees. The femoral head was seated freely in a cup made of polyoxymethylene $\triangle \mathrm{POM} \otimes$, which simulates the function of the acetabulum.

\section{Biomechanical testing}

\section{Axial load testing}

Before the official axial load test, the models were preloaded under a velocity of $5 \mathrm{~mm} / \mathrm{min}$ and the maximum load of $100 \mathrm{~N}$ to stabilize the construct. Then, the models were loaded in axial compression of $5 \mathrm{~mm} / \mathrm{min}$ from the base line, and the test was stopped when it reached $1400 \mathrm{~N}$ and before any fixation failure was visualized (Figure 3a). The axial stiffness was calculated (axial stiffness equal to axial load divided by displacement).

\section{Torsion testing}

The torsion test was performed after the axial load test. The femur was stabilized proximally and distally by a self-made holder $(10 \times 1010 \mathrm{~cm})$. We conducted the torsion in the control mode of $1.0 \mathrm{~Hz}$. The torque was recorded at the points of $0,1,2,3,4$, and 5 degrees (Figure $3 b$ ), and the torsion stiffness was calculated respectively (torsion stiffness equal to torque divided by torsion degree).

\section{Axial load-to-failure testing}


After the axial load and torsion test, if there were no failure of the construct's fixation, it was subject to an axial load-to-failure test. The load mode was kept the same as in the axial loading test, and the implantfemur constructs were positioned in a single-leg stance under the axial compression at the rate of 5 $\mathrm{mm} / \mathrm{min}$ (Figure 3c). Failure was defined as the one of the following: 1) a new fracture line was found in model; 2) failure of internal fixation, such as bending or breakage of plates or screws; 3) $15 \mathrm{~mm}$ of actuator displacement; or 4) the load-displacement curve became a flat curve, indicating that there was no change of axial load when displacement 161 increased. All constructs of failure load were recorded.

\section{Statistics}

All statistical calculations and analysis were performed by SPSS version 21.0 software (SPSS Inc., Chicago, IL, USA). All statistics were performed by mean \pm standard deviation ( $x \pm s)$. One-way analyses of variance (ANOVA) was used for comparison among groups, and the Student-Newman-Keuls (SNK) test was used for comparison between any two means. The level of statistical significance was defined as $\mathrm{p}<0.05$.

\section{Results}

\section{Axial load testing}

All axial load data were collected in a load-displacement curve, with slope equal to the axial stiffness's reciprocal value, and we found the linear curve in the four groups from 0 to $1000 \mathrm{~N}$. After the axial load reached a level over $1000 \mathrm{~N}$, the axial stiffness of the PFNA and PFLCP group reduced, whereas the medial buttress plate and bilateral plate group increased slowly. From 0 to $600 \mathrm{~N}$, the medial buttress plate's stiffness showed almost no difference with that of the bilateral plate group, whereas the PFNA group and the lateral plate group showed the same trend from 0 to $400 \mathrm{~N}$ (Figure 4). In our analysis, all data met homogeneity of variances. The mean axial stiffness and standard deviation of the PFLCP group were $109.42 \pm 30.14 \mathrm{~N} / \mathrm{mm}, 119.13 \pm 29.14 \mathrm{~N} / \mathrm{mm}$ for the PFNA group, $162.05 \pm 22.05 \mathrm{~N} / \mathrm{mm}$ for the PFMBP group, and $178.64 \pm 32.93 \mathrm{~N} / \mathrm{mm}$ for the PFLCP+PFMBP group. There was a statistical difference between each of the four groups when compared with one another $(P<0.05)$ (Figure 5$)$.

\section{Torsion testing}

The torque increased with increasing degree of torsion in the four groups. The torque resulting from different degrees of torsion in the same group showed significant differences $(P<0.05)$; moreover, torque of the same degree in the four groups showed significant difference $(P<0.05)$ (Table 1). The torsion stiffness in PFLCP was $(1.45 \pm 0.44) \mathrm{N} \times \mathrm{mm} / \mathrm{deg},(1.10 \pm 0.13) \mathrm{N} \times \mathrm{mm} / \mathrm{deg}$ in the PFNA group, $(1.45 \pm 0.18) \mathrm{N} \times \mathrm{mm} / \mathrm{deg}$ in the PFLCP+PFMBP group, and $(1.36 \pm 0.32) \mathrm{N} \times \mathrm{mm} / \mathrm{deg}$ in the PFMBP group (Figure 6). All data met homogeneity of variances, and there was a significant difference between the four groups with respect to torsion stiffness $(P<0.05)$. Nevertheless, there was no significant difference 
between the PFLCP, PFMBP, and PFLCP+PFMBP groups $(P>0.05)$, and these three groups showed a significant difference with the PFNA group $(P<0.05)$.

\section{Axial load-to-failure testing}

There were no plates or screws broken or bended in any of the groups. In the PFLCP group, we found one case of a new fracture that occurred, and the remaining five cases had proximal fracture fragment displacement. Two cases in the PFNA group had a new fracture in the distal locking screw area, and four cases had proximal fracture fragment displacement. Every case in the bilateral plate group had actuator displacement of $15 \mathrm{~mm}$. In the PFMBP group, there were three cases of new fracture in the distal area of the plate, and three cases of actuator displacement of $15 \mathrm{~mm}$ (Figure 7). The axial load-to-failure in the PFLCP group was $1408.88 \pm 0.17 \mathrm{~N}, 1696.56 \pm 0.52 \mathrm{~N}$ for the PFNA group, $2283.35 \pm 0.46 \mathrm{~N}$ for the PFLCP+PFMBP group, and $2154.65 \pm 0.10 \mathrm{~N}$ for the PFMBP group (Figure 8). The axial load-to-failure in the four groups was significantly different $(P<0.05)$.

\section{Discussion}

Reverse oblique intertrochanteric fracture, an unstable type of intertrochanteric fracture, can cause morbidity and mortality. The incidence of this fracture is increasing with an aging population in developed and developing countries. The majority of surgeons choose an intramedullary device to treat reverse oblique intertrochanteric fractures because of its superior stable fixation [9]. PFNA was first developed by the AO/ASIF in 2004, and its characteristic of the use of an anti-rotation blade provides stability when inserted into cancellous bone. The shape of the PFNA provides increased contact between implant and bone, and its anti-rotation blade in the femoral head decreases the incidence of rotation and cutout compared to traditional screws [10]. The primary challenge for fixing a reverse oblique intertrochanteric fracture is to overcome shear force along the fracture line. PFNA's anti-rotation blade in the femoral head and nail in the medullary resist proximal fracture fragment sliding laterally and distal fragment sliding medially. With the wide use of the PFNA, the fixation failure rate has also increased. Failure often results from the blade inserting into the hip joint, the blade dropping out, coxa vara, and proximal femoral splitting fracture. The failure rate was reported to be $3 \%$ to $40 \%[11,12]$, and typically caused by improper operation, unstable fracture, and osteoporosis. The PFLCP was an alternative method for proximal femoral fracture, and is one of the most widely used extramedullary implants. According to some literature [13-15], the PFLCP also has a failure rate of $37 \%$ to $40 \%$. There were various biomechanical studies [16-18] comparing intramedullary versus extramedullary devices to treat intertrochanteric fractures; from these studies, we found that intramedullary devices were superior to extramedullary devices in terms of biomechanical properties. A series of clinical studies reported the intramedullary device's superiority in less surgery damage, stable fixation, and fewer complications when compared to extramedullary implants [9,19-21]. One biomechanical study [22] compared PFLCP and PFNA for reverse oblique intertrochanteric fractures; however, to the best of our knowledge, no comparative study of PFNA, PFLCP, medial buttress plate, and bilateral plate exists. 
Our newly designed medial buttress plate changed the tradition of extramedullary device planted laterally, as the anatomic plate could fit the femoral neck well. We can say that the medial buttress plate has become an innovation, our anatomic plate was firstly used in treating unstable femoral intertrochanteric fractures. Since Mir H. et al [23] hypothesized that the use of the medial buttress plate may prevent fixation failure in vertical femoral neck fractures. Chang. et al [24] also emphasized the importance of positive medial cortical support for unstable pertrochanteric fractures. At the same time, the primary question is, which surgical approach best suits the medial buttress plate? The lateral approach, a commonly used extramedullary approach for intertrochanteric fractures, could provide direct access to the vastus lateralis muscle; however, it has the disadvantage of limited exposure, long surgery time, and substantial bleeding. The direct anterior approach (DAA), first described by Hunter [25], is a modified Smith-Peterson approach with less soft-tissue damage, shorter surgery duration, and less bleeding than traditional lateral and posterior-lateral approaches [26]. However, DAA has some drawbacks such as a long learning curve [27], difficulty in femur exposure, and the possibility of lateral femoral cutaneous nerve (LFCN) and lateral femoral circumflex artery (LFCA) injury. After reviewing a series of literature, we found an improved approach proposed by Li. et al[8]: the anterolateral intermuscular approach. The anterolateral intermuscular approach exposes the femoral neck and proximal femoral intertrochanteric based on conventional space between the gluteus medius and the tensor fasciae latae. Initial clinical studies confirmed that this approach is relatively safe, provides excellent exposure, and causes less softtissue damage than the traditional approach $[28,29]$. We believe that it represents a promising new method to surgically treat reverse oblique intertrochanteric fractures.

In our biomechanical research, PFLCP+PFMBP had the highest axial stiffness of the four groups, whereas PFLCP achieved lowest. In the other two groups, PFMBP showed superior axial stiffness to PFNA. Forward. et al[30]in his study stated that intramedullary nail achieved the best axial stiffness when compared to locking plate and 95-degree angled blade plate. Bong. et al[31]confirmed intramedullary had an advantage over sliding hip screw in stiffness. We found that the medial buttress plate had better performance than PFNA for these reasons: 1) The thickness of the medial buttress plate was $8 \mathrm{~mm}$, whereas the diameter of PFNA was $9 \mathrm{~mm}$ on average and PFLCP was $5 \mathrm{~mm}$; the thickness of PFNA and medial buttress plate is greater than PFLCP, making the stiffness of PFNA and medial buttress plate greater than that of PFLCP. 2) The connect area of the proximal and distal plates in PFMBP is short and wide, which results in less arm of force and better mechanical stability. 3) The proximal part of PFMBP was designed to have much more contact area between the femoral neck and the implant, which may be a reason for superior medial buttress performance. 4) Two lag screws offer efficient pressure on fragment. In load-to-failure testing, the PFLCP+PFMBP and the PFMBP had higher failure load than did the PFNA, which proved that our medial buttress plate has stronger stiffness in axial load. Fensky. et al [32] reported that intramedullary and extramedullary showed no significant difference in torsion stiffness. Rupprecht. et al [33] verified that torsion stiffness showed no difference between intra- and extramedullary in femoral neck fracture. Our torsion testing showed that there was no significant difference between the PFLCP, PFMBP, and PFLCP+PFMBP groups; the PFNA was different with respect to torsion stiffness. We considered that our results of higher torsion stiffness in the medial buttress over the 
PFLCP and PFNA was mainly due to our design. The proximal part of the plate uses three screws, while the lateral two screws are designed to toe-out. As with PFLCP, more screws in the distal part could add holding force on the femoral head, increasing the torsion stiffness. On the basis of these theories, PFLCP+PFMBP should have achieved better axial load and rotation, and our experiment has proved it.

The specimen chosen, Synbone, is made of rigid polyurethane foam and simulates human cancellous bone [34]. Some researchers had already used synthetic bone in their studies. Luo. et al [35] used Synbone to compare helical blade with screw design for sliding hip fixations, and Marmor [36] used Sawbone to compare the influence of different lengths of intramedullary nails in fixed intertrochanteric fracture. There were still some limitations to our study. First, the absence of soft tissue and ligaments from synthetic bone could not simulate clinical conditions in the real human body. Second, because Sawbone surrogates were much better than Synbone surrogates to real bone, we will use Sawbone to perform biomechanical studies in the future. Third, by this approach, the fracture obtained an anatomic reduction as would a traditional extramedullary implant. However, this implant would be an intracapsular implant. The reconstruction period may destroy the blood supply of the capsular, and increase the risk of avascular necrosis of the femur head (ANFH) and nonunion of the fracture. Thus, an anatomic study should be conducted to confirm whether intracapsular injury exists. Fourth, insufficient biomechanical indicators concluded only axial load stiffness and torsion stiffness; further studies should involve tests such as the bending test and a fatigue load test. Our biomechanical study could not reflect actual fracture fixation properties, yet effective information has been provided for comparing relative stabilities in four fixation methods.

\section{Conclusion}

In summary, our newly designed medial buttress plate achieved excellent stiffness in axial load and torsion compared to PFNA and PFLCP to fix reverse oblique intertrochanteric fractures in biomechanical research, whereas the bilateral plate showed better stiffness than PFMBP. The research also indicated that the implant may have a good outcome in treating reverse oblique intertochanteric fractures because of its performance under medial compressive stress. Future research should focus on large-size samples, with sufficient testing of mechanical properties in proven clinical settings.

\section{Abbreviations}

PFNA囚Proximal Femoral Nail Anti-rotation; PFMBP: Proximal femoral medial buttress plate; PFLCP: Proximal femoral locking compression plate; OTA: Orthopaedic Trauma Association; ER: Ethoxyline resin; POM: Polyoxymethylene; ANOVA: One-way analyses of variance; SNK: Student-Newman-Keuls; DAA:

Direct anterior approach; LFCN: Lateral femoral cutaneous nerve; ANFH: Avascular necrosis of the femur head.

\section{Declarations}


Ethics approval and consent to participate

Not applicable

Consent to publish

Not applicable

Availability of data and materials

We do not wish to share our data, because some of data regarding novel implant secret.

Competing interests

The author(s) declared no potential conflicts of interest with respect to the research, authorship, and/or publication of this article.

Funding

Not applicable

Authors' Contributions

RZ contributed to the study design, the study management and critical revision of the manuscript. RZ contributed to the primary manuscript drafting. KYW, PL and XSG contributed to data collection and data analysis.

Acknowledgements

Not applicable

\section{References}

[1]. Park SY, Yang KH, Yoo JH, Yoon HK and Park HW. The treatment of reverse obliquity intertrochanteric fractures with the intramedullary hip nail. J Trauma 2008; 65: 852-857.

[2]. Yang X, Wu Q and Wang X. Investigation of perioperative hidden blood loss of unstable intertrochanteric fracture in the elderly treated with different intramedullary fixations. Injury $2017 ; 48$ : $1848-1852$.

[3]. Guo Y, Yang HP, Dou QJ, He XB and Yang XF. Efficacy of femoral nail anti-rotation of helical blade in unstable intertrochanteric fracture. Eur Rev Med Pharmacol Sci 2017; 21: 6-11.

[4]. Haidukewych GJ, Israel TA and Berry DJ. Reverse obliquity fractures of the intertrochanteric region of the femur. J Bone Joint Surg Am 2001; 83-A: 643-650. 
[5].Matre K, Havelin LI, Gjertsen JE, Vinje T, Espehaug B and Fevang JM. Sliding hip screw versus IM nail in reverse oblique trochanteric and subtrochanteric fractures. A study of 2716 patients in the Norwegian Hip Fracture Register. Injury 2013; 44: 735-742.

[6]. Yadav S, Sinha S, Luther E, Arora NC, Prasad M and Varma R. Comparison of extramedullary and intramedullary devices for treatment of subtrochanteric femoral fractures at tertiary level center. Chin $\mathrm{J}$ Traumatol 2014; 17: 141-145.

[7]. Sun D, Park BS, Jang GI and Lee B. The Fixation Method according to the Fracture Type of the Greater Trochanter in Unstable Intertrochanteric Fractures Undergoing Arthroplasty. Hip Pelvis 2017; 29: 62-67.

[8]. Li B, Zhang B, Ding Z, Liu Y and Dai M. Anterolateral intermuscular approach for type A2 intertrochanteric fractures: a cadaveric study. Int Surg 2015; 100: 314-319.

[9]. Simmermacher RK, Ljungqvist J, Bail H, Hockertz T, Vochteloo AJ, Ochs U, Werken C and studygroup AP. The new proximal femoral nail antirotation (PFNA) in daily practice: results of a multicentre clinical study. Injury 2008; 39: 932-939.

[10]. Soucanye de Landevoisin E, Bertani A, Candoni P, Charpail C and Demortiere E. Proximal femoral nail antirotation (PFN-ATM) fixation of extra-capsular proximal femoral fractures in the elderly: retrospective study in 102 patients. Orthop Traumatol Surg Res 2012; 98: 288-295.

[11]. Makki D, Matar HE, Jacob N, Lipscombe S and Gudena R. Comparison of the reconstruction trochanteric antigrade nail (TAN) with the proximal femoral nail antirotation (PFNA) in the management of reverse oblique intertrochanteric hip fractures. Injury 2015; 46: 2389-2393.

[12]. Chou DT, Taylor AM, Boulton C and Moran CG. Reverse oblique intertrochanteric femoral fractures treated with the intramedullary hip screw (IMHS). Injury 2012; 43: 817-821.

[13]. Wirtz C, Abbassi F, Evangelopoulos DS, Kohl S, Siebenrock KA and Kruger A. High failure rate of trochanteric fracture osteosynthesis with proximal femoral locking compression plate. Injury 2013; 44: 751-756.

[14]. Streubel PN, Moustoukas MJ and Obremskey WT. Mechanical failure after locking plate fixation of unstable intertrochanteric femur fractures. J Orthop Trauma 2013; 27: 22-28.

[15]. Lin SJ, Huang KC, Chuang PY, Lee CY, Huang TW, Lee MS and Hsu RW. The outcome of unstable proximal femoral fracture treated with reverse LISS plates. Injury 2016; 47: 2161-2168.

[16]. Kuzyk PR, Lobo J, Whelan D, Zdero R, McKee MD and Schemitsch EH. Biomechanical evaluation of extramedullary versus intramedullary fixation for reverse obliquity intertrochanteric fractures. $J$ Orthop Trauma 2009; 23: 31-38. 
[17]. Weiser L, Ruppel AA, Nuchtern JV, Sellenschloh K, Zeichen J, Puschel K, Morlock MM and Lehmann W. Extra- vs. intramedullary treatment of pertrochanteric fractures: a biomechanical in vitro study comparing dynamic hip screw and intramedullary nail. Arch Orthop Trauma Surg 2015; 135: 1101-1106.

[18]. Knobe M, Gradl G, Buecking B, Gackstatter S, Sonmez TT, Ghassemi A, Stromps JP, Prescher A and Pape HC. Locked minimally invasive plating versus fourth generation nailing in the treatment of AO/OTA 31A2.2 fractures: A biomechanical comparison of $\operatorname{PCCP}((R))$ and Intertan nail((R)). Injury 2015; 46:

$1475-1482$.

[19]. Evaniew N and Bhandari M. Cochrane in CORR (R): Intramedullary nails for extracapsular hip fractures in adults (review). Clin Orthop Relat Res 2015; 473: 767-774.

[20]. Makki D, Matar HE, Jacob N, Lipscombe S and Gudena R. Comparison of the reconstruction trochanteric antigrade nail (TAN) with the proximal femoral nail antirotation (PFNA) in the management of reverse oblique intertrochanteric hip fractures. Injury 2015; 46: 2389-2393.

[21]. Irgit K, Richard RD, Beebe MJ, Bowen TR, Kubiak E and Horwitz DS. Reverse Oblique and Transverse Intertrochanteric Femoral Fractures Treated With the Long Cephalomedullary Nail. J Orthop Trauma 2015; 29: e299-304.

[22]. Ma JX, Wang J, Xu WG, Yu JT, Yang Y and Ma XL. Biomechanical outcome of proximal femoral nail antirotation is superior to proximal femoral locking compression plate for reverse oblique intertrochanteric fractures: a biomechanical study of intertrochanteric fractures. Acta Orthop Traumatol Turc 2015; 49: 426-432.

[23]. Mir $\mathrm{H}$ and Collinge $\mathrm{C}$. Application of a medial buttress plate may prevent many treatment failures seen after fixation of vertical femoral neck fractures in young adults. Med Hypotheses 2015; 84: 429433.

[24]. Chang SM, Zhang YQ, Ma Z, Li Q, Dargel J and Eysel P. Fracture reduction with positive medial cortical support: a key element in stability reconstruction for the unstable pertrochanteric hip fractures. Arch Orthop Trauma Surg 2015; 135: 811-818.

[25]. Rachbauer F, Kain MS and Leunig M. The history of the anterior approach to the hip. Orthop Clin North Am 2009; 40: 311-320.

[26]. Amanatullah DF, Masini MA, Roger DJ and Pagnano MW. Greater inadvertent muscle damage in direct anterior approach when compared with the direct superior approach for total hip arthroplasty. Bone Joint J 2016; 98-B: 1036-1042.

[27]. de Steiger RN, Lorimer M and Solomon M. What is the learning curve for the anterior approach for total hip arthroplasty? Clin Orthop Relat Res 2015; 473: 3860-3866. 
[28]. Wu B, Dai M, Zou F, Zhang B, Li B, Luo S and Qiu P. [Effectivenes of anterolateral C-shaped approach in treatment of intertrochanteric fracture of femur in elderly patient]. Zhongguo Xiu Fu Chong Jian Wai Ke Za Zhi 2013; 27: 985-989.

[29]. Dai M, Zou F, Zhang B, Nie T, Cheng M, Zou Y and Jiang C. [Effectiveness comparison of improved anterolateral approach and lateral approach in treatment of senile intertrochanteric fracture]. Zhongguo Xiu Fu Chong Jian Wai Ke Za Zhi 2012; 26: 910-914.

[30]. Forward DP, Doro CJ, O'Toole RV, Kim H, Floyd JC, Sciadini MF, Turen CH, Hsieh AH and Nascone JW. A biomechanical comparison of a locking plate, a nail, and a 95 degrees angled blade plate for fixation of subtrochanteric femoral fractures. J Orthop Trauma 2012; 26: 334-340.

[31]. Bong MR, Patel V, lesaka K, Egol KA, Kummer FJ and Koval KJ. Comparison of a sliding hip screw with a trochanteric lateral support plate to an intramedullary hip screw for fixation of unstable intertrochanteric hip fractures: a cadaver study. J Trauma 2004; 56: 791-794.

[32]. Fensky F, Nuchtern JV, Kolb JP, Huber S, Rupprecht M, Jauch SY, Sellenschloh K, Puschel K, Morlock MM, Rueger JM and Lehmann W. Cement augmentation of the proximal femoral nail antirotation for the treatment of osteoporotic pertrochanteric fractures-a biomechanical cadaver study. Injury 2013; 44: 802-807.

[33]. Rupprecht M, Grossterlinden L, Ruecker AH, de Oliveira AN, Sellenschloh K, Nuchtern J, Puschel K, Morlock M, Rueger JM and Lehmann W. A comparative biomechanical analysis of fixation devices for unstable femoral neck fractures: the Intertan versus cannulated screws or a dynamic hip screw. J Trauma 2011; 71: 625-634.

[34]. O'Neill F, Condon F, McGloughlin T, Lenehan B, Coffey JC and Walsh M. Dynamic hip screw versus DHS blade: a biomechanical comparison of the fixation achieved by each implant in bone. J Bone Joint Surg Br 2011; 93: 616-621.

[35]. Luo Q, Yuen G, Lau TW, Yeung K and Leung F. A biomechanical study comparing helical blade with screw design for sliding hip fixations of unstable intertrochanteric fractures. ScientificWorldJournal 2013; 2013: 351936.

[36]. Marmor M, Elliott IS, Marshall ST, Yacoubian SV, Yacoubian SV and Herfat ST. Biomechanical comparison of long, short, and extended-short nail construct for femoral intertrochanteric fractures. Injury 2015; 46: 963-969.

\section{Tables}

Table 1 Comparison of torque corresponding to the torsion angles between four groups $(\mathrm{N} \times \mathrm{mm}, \mathrm{x} \pm \mathrm{s})$ 


\begin{tabular}{llllllll}
\hline \hline Group & $0^{\circ}$ & $1^{\circ}$ & $2^{\circ}$ & $3^{\circ}$ & $4^{\circ}$ & $5^{\circ}$ & Statistics \\
\hline PFNA & 0 & $1.09 \pm 0.02$ & $2.16 \pm 0.01$ & $3.28 \pm 0.11$ & $4.33 \pm 0.03$ & $5.47 \pm 0.02$ & $F=98.89$ \\
& & & & & & & $P=0.000$ \\
PFMBP & 0 & $1.46 \pm 0.04$ & $2.62 \pm 0.09$ & $3.97 \pm 0.01$ & $5.01 \pm 0.03$ & $6.06 \pm 0.04$ & $F=75.60$ \\
& & & & & & & $P=0.000$ \\
PFLCP & 0 & $1.48 \pm 0.09$ & $2.67 \pm 0.03$ & $3.93 \pm 0.02$ & $5.36 \pm 0.04$ & $6.51 \pm 0.05$ & $F=76.90$ \\
& & & & & & & $P=0.000$ \\
PFLCP+PFMBP & 0 & $1.56 \pm 0.01$ & $2.87 \pm 0.01$ & $4.15 \pm 0.04$ & $5.23 \pm 0.02$ & $6.41 \pm 0.01$ & $F=90.74$ \\
& & & & & & & $P=0.000$ \\
Statistics & - & $F=85.97$ & $F=78.43$ & $F=79.69$ & $F=91.27$ & $F=84.57$ & \\
& & & & & & & \\
& & & & & & & \\
\end{tabular}

\section{Figures}

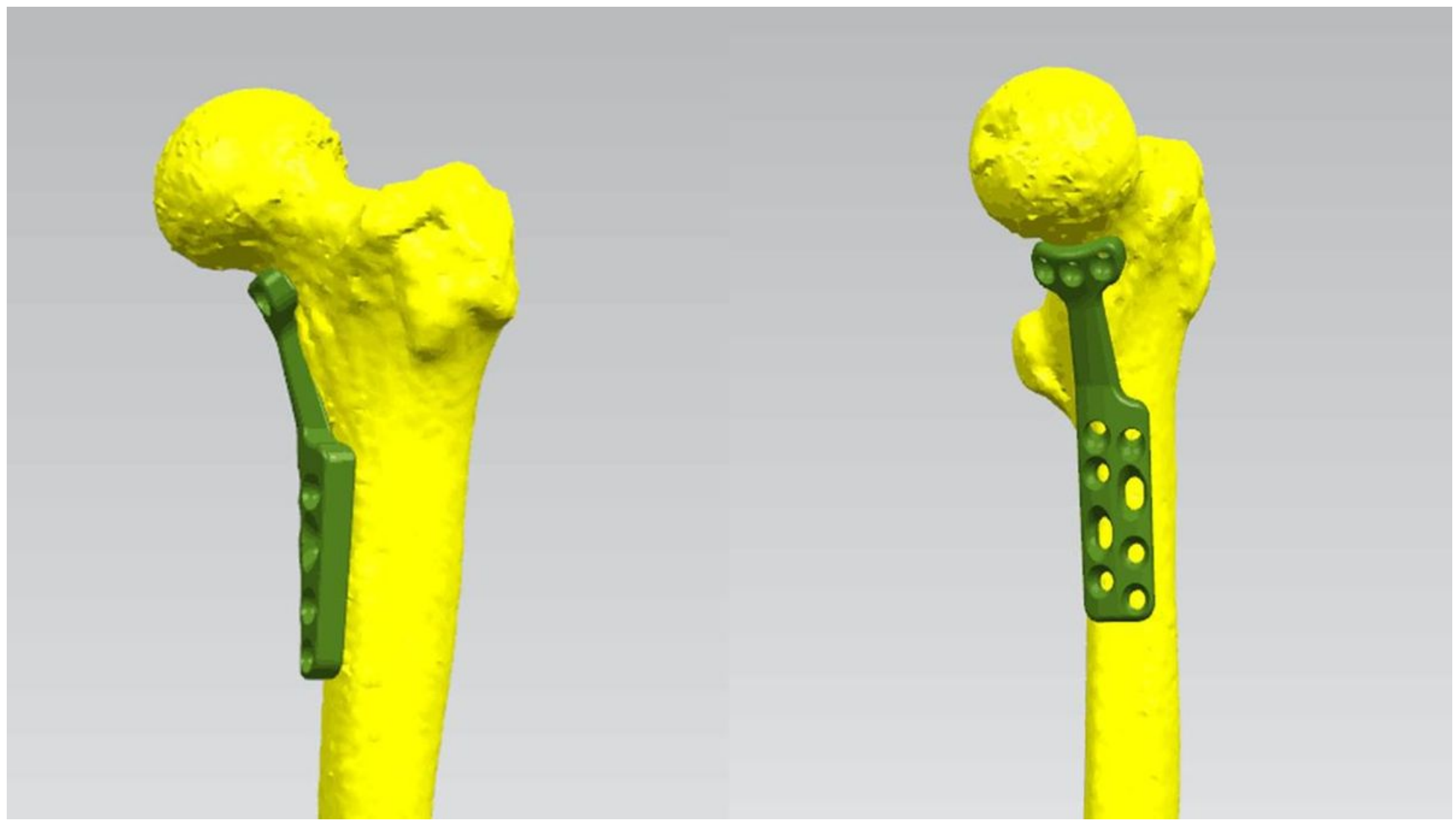

\section{Figure 1}

Our newly designed T-shaped medial buttress plate has three holes proximally and eight holes distally. Two lag screws could be planted in the medial of the intertrochanteric through two distal holes. 


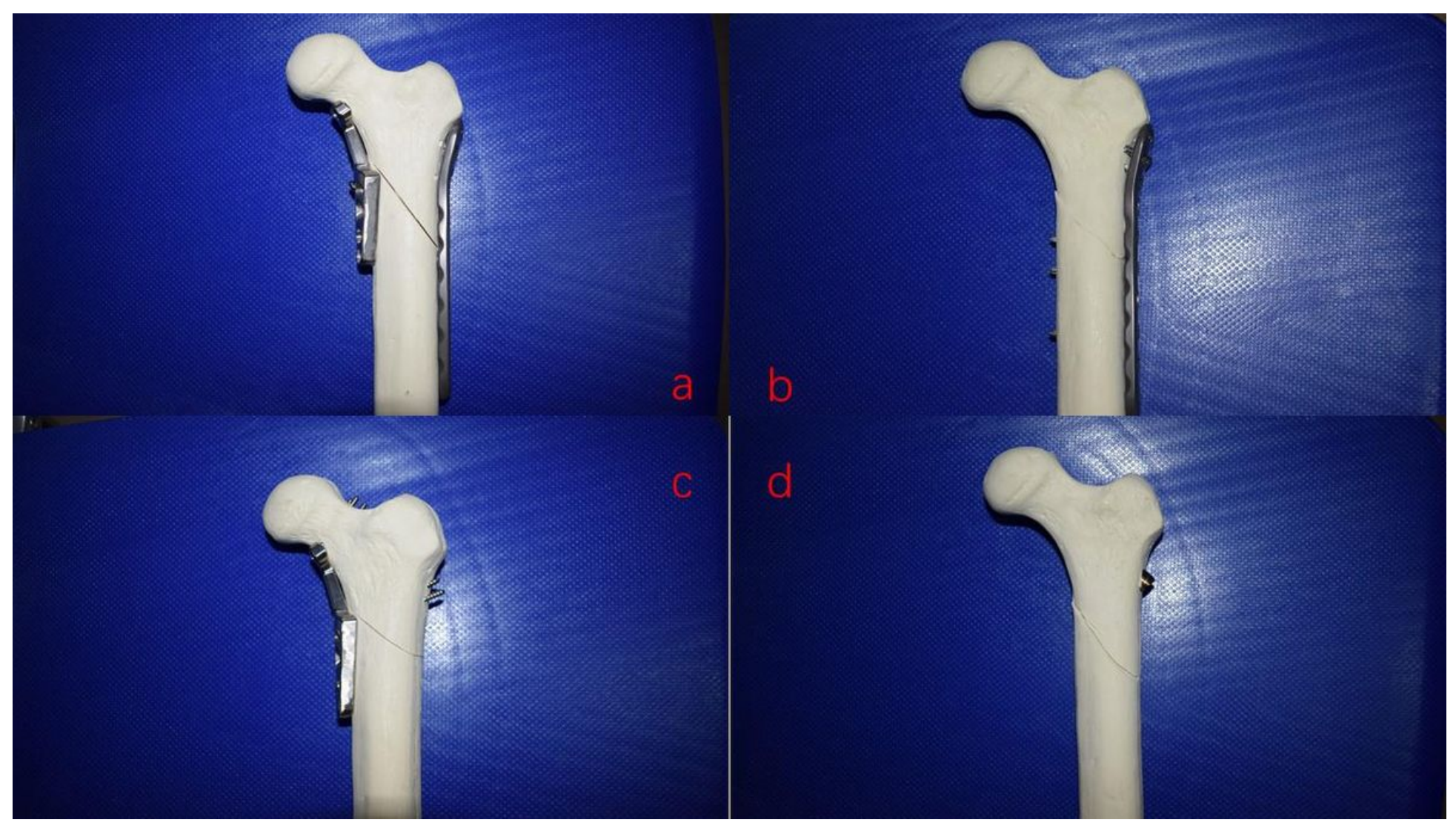

Figure 2

The fracture model in our biomechanical testing: a) PFLCP+PFMBP; b) PFLCP; c) PFMBP; d) PFNA.

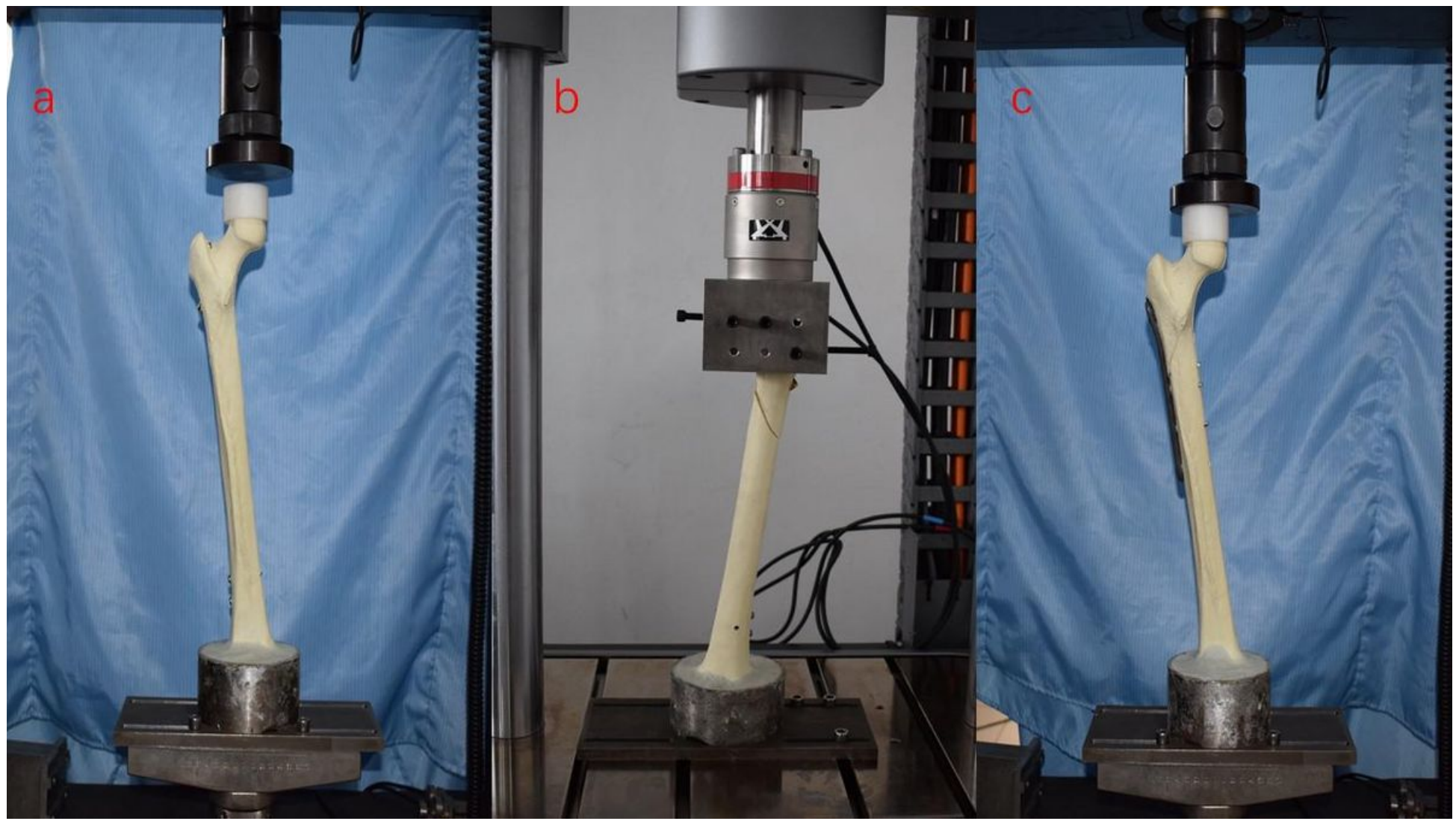


Figure 3

Biomechanical testing modes: a) axial load testing; b) torsion testing; c) axial load-to-failure testing.

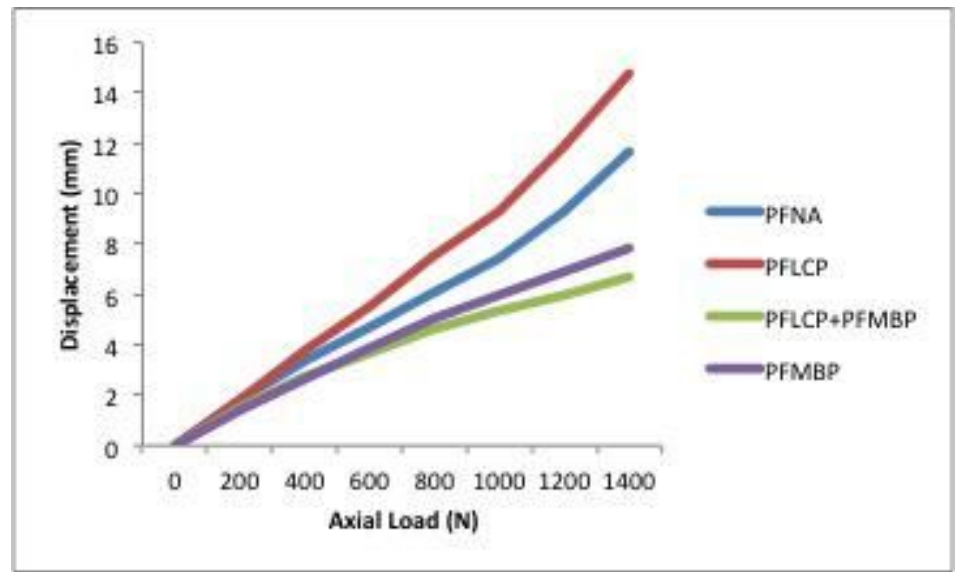

Figure 4

Axial load-displacement curve in the four groups.
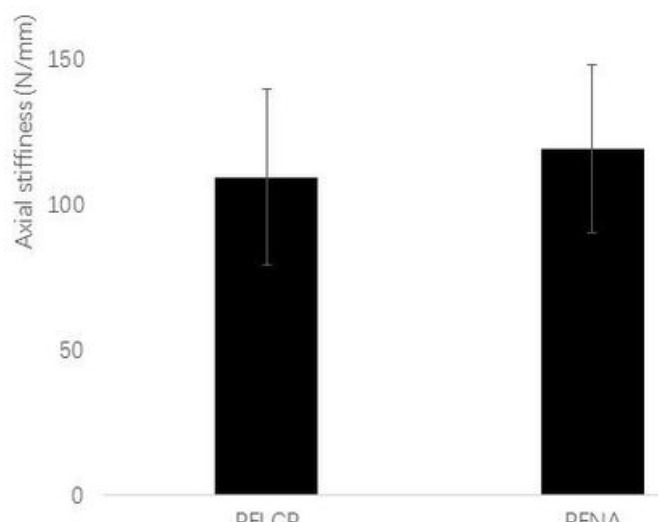

PFNA

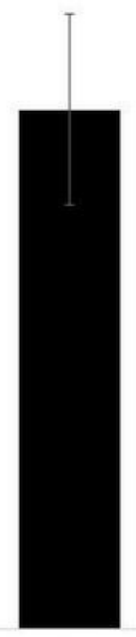

PFLCP +PFMBP

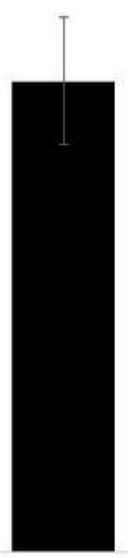

PFMBP

Figure 5

Stiffness in axial load between the four groups 


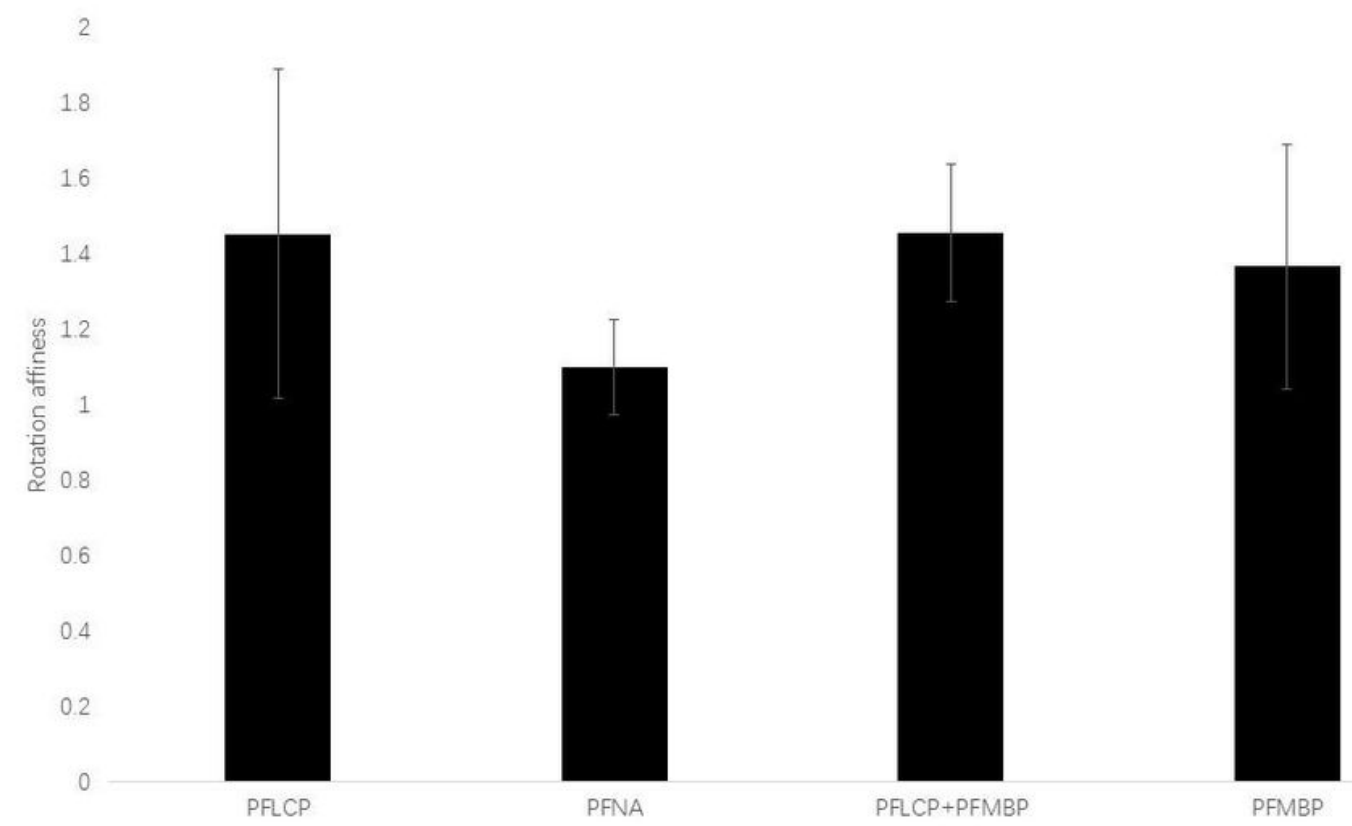

Figure 6

Stiffness in torsion between the four groups

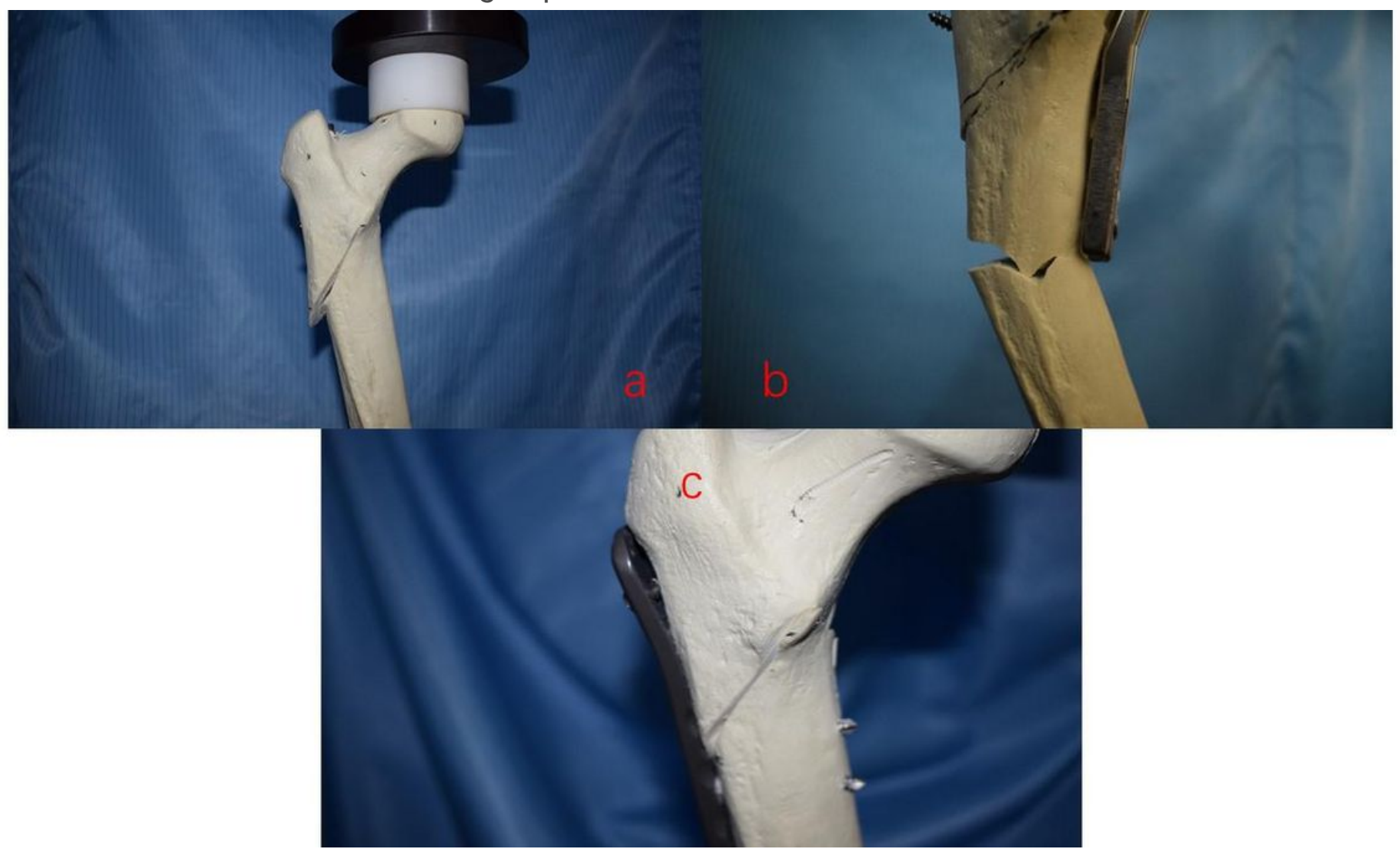


Figure 7

Failure mode for constructs: a) proximal fracture fragment displacement in the PFNA group; $b$ ) new fracture in distal area of plate in the PFMBP group; c) proximal fracture fragment displacement in the PFLCP group.

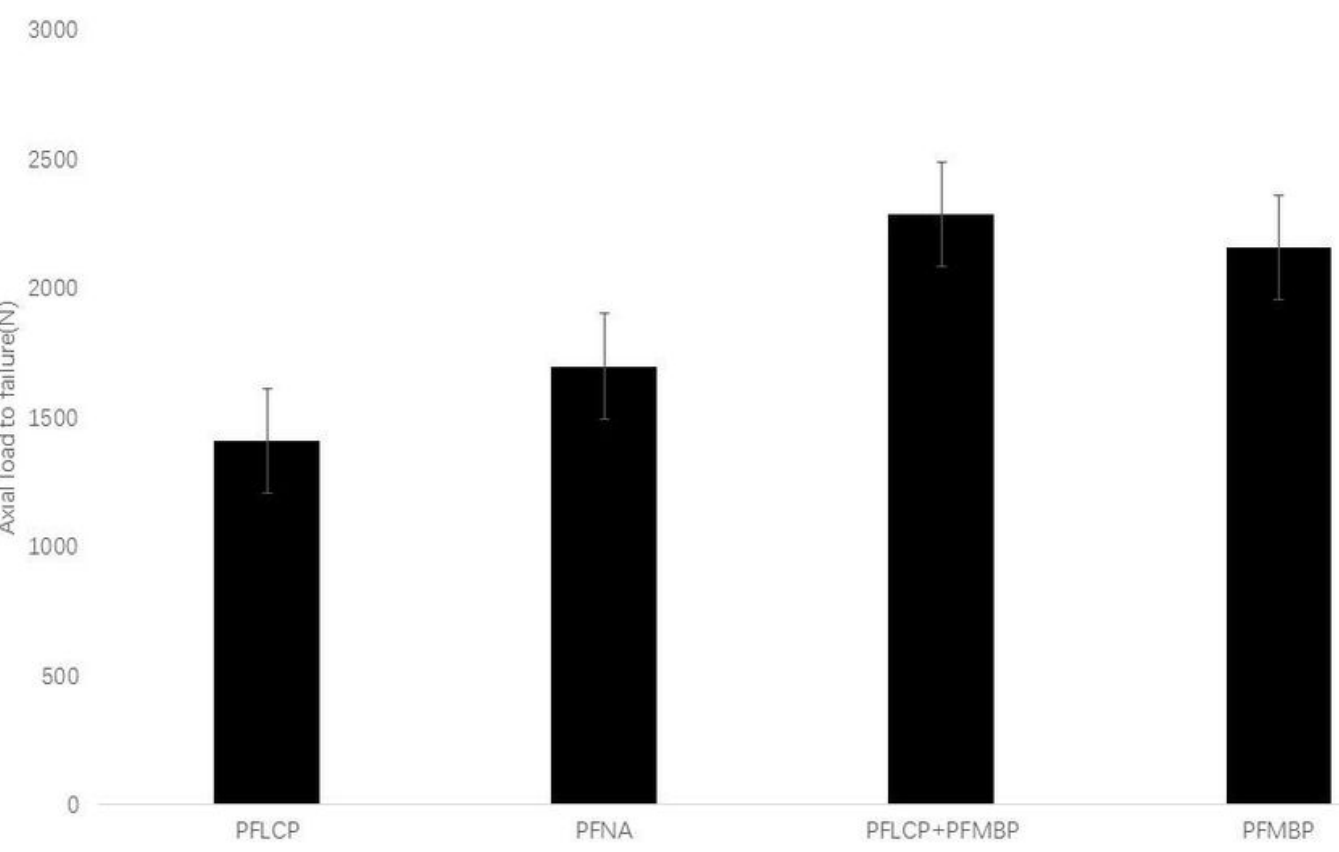

Figure 8

Failure in axial load between the four groups. 